\title{
Tail Risk Early Warning System for Capital Markets Based on Machine Learning Algorithms
}

\author{
Zongxin Zhang ${ }^{1} \cdot$ Ying Chen $^{1}$ (1)
}

Accepted: 20 July 2021 / Published online: 28 July 2021

(C) The Author(s), under exclusive licence to Springer Science+Business Media, LLC, part of Springer Nature 2021

\begin{abstract}
Scientific and effective tail risk measurement and early warning are key points and difficulties in the identification and control of major risks in capital markets. In this paper, we use the autoregressive conditional Fréchet model (AcF) to construct a tail risk measurement index for the capital market in China. The tail risk status identified by the scientific index method is used as a monitoring anchor to construct and optimize a tail risk early warning model based on machine learning algorithms. The study yields three findings. (1) The AcF model can overcome the shortcomings of traditional models in tail risk measurement and significantly improve the tail risk measurement efficiency of the capital market. (2) Tail risk synergies between equity and bond markets are significantly stronger than yield synergies, and the tail risk measure index has the role of a leading indicator of significant risk in capital markets. (3) Based on the joint test of risk status and crisis identification efficiency, the Logit model of crisis identification fails whereas the tail risk warning model optimized by machine learning algorithms can accurately identify crises and significant risks. The optimal early warning model pairings for the stock market and bond market are the oversampling-random forest algorithm and the double sampling-random forest algorithm, respectively, with out-of-sample crisis warning accuracies of $81.94 \%$ and $90.20 \%$, respectively.
\end{abstract}

Keywords Tail Risk Measurement · Risk Warning $\cdot$ AcF Model · Machine Learning Algorithms

Ying Chen

chenying18@fudan.edu.cn

1 School of Economics, Fudan University, Shanghai, China 


\section{Introduction}

Existing research and international experience suggest that market sentiment and cross-market risk correlation channels can amplify the negative impact of extreme events following tail risk exposures in capital markets. Risks are contagious and spread throughout the financial system, leading to systemic financial crises and significant losses for market participants (Benoit et al., 2017; Billio et al., 2012). As a result of the Sino-US trade war and the COVID-19 pandemic, the current international economic order is in deep adjustment, and the financial system has shown extremely high sensitivity to external shocks as abnormal market volatility exacerbates the endogenous vulnerability of the financial system ( $\mathrm{Li}$ et al., 2020; Yang et al., 2020). These circumstances have fostered an accumulation of incremental risks in the financial market and a continuous escalation of stock risk pressure. As a leading indicator of systemic financial risk, the measuring and forecasting of tail risk and its early warning signal are important to the systemic risk control system of the capital market. In view of the increasingly complex financial market system, it is of great academic value and practical significance to develop a sound tail risk measurement method for the capital market and to improve the efficiency of tail risk monitoring.

Regarding prior research on tail risk measures and synergies, based on the value at risk (VaR) measurement of market risk (Engle, 2002; Engle \& Manganelli, 2004) implemented tail risk measures and analyzed cross-market synergies using quantile regression. Subsequently, Girardi and Ergün (2013) proposed the generalized autoregressive conditional heteroscedastic conditional value at risk (GARCH-CoVaR) method to characterize the tail risk contagion effect in financial markets. The VaR family model gradually became the mainstream model for tail risk studies. The model is also widely used in relation to the Chinese capital market (Li et al., 2010; Xu \& Huang, 2008). Furthermore, the application and extension of quantile regression-based CoVaR models have further enriched the research on systematic risk measurement and cross-market contagion (Adrian \& Brunnermeier, 2016). Although VaR family models have made important contributions to tail risk research, financial time series with spiky and thick-tailed distributions pose challenges of non-normality. Moreover, the GARCH-VaR models measure risk based on current volatility and have insufficient foresight in relation to extreme events (Longin, 2000).

In order to improve the efficiency of tail risk measurement and early warning, extreme value theory was introduced. The distribution characterized by extreme values is sufficient to study the thick-tailed distribution (Jansen \& Vries, 1991). Then, the generalized extreme value distribution and the generalized Pareto distribution were developed successively, which correspond to the extreme value modeling of the block extreme value (Embrechts \& Schmidli, 1994) and the peak crossing threshold method (McNeil, 1998).

Extreme value theory relaxes the assumption of a priori distribution of the data and has better applicability for financial time series of a thick-tailed nature. Static extreme value theory was used to measure the tail risk of the Chinese stock market, 
verifying its effectiveness in the field of tail risk measurement of the Chinese capital market (Feng, 2002; Wang \& Zhou, 2012). However, because of its inability to directly portray the dynamic changes of tail risk, scholars have further extended static extreme value theory to a dynamic framework such as the dynamic peak crossing threshold method (Massacci, 2017) or the autoregressive conditional Fréchet (AcF) model (Zhao et al., 2018). Compared with the dynamic peak crossing threshold method, the AcF model achieves dynamic measurement of tail risk while directly modeling the outliers, which minimizes the information loss. In addition, this approach reduces the estimation bias incurred by the model threshold selection. Thus, the estimation efficiency of the model is further improved.

Financial risk identification, monitoring, and early warning are interlinked in the risk control system. The objective of risk measurement is to serve macro-prudential management. Informing future risk based on market risk measurement and early warning is what financial risk management is seeking. The construction and optimization of the risk warning model is an important prerequisite for effective risk research and control. The main representatives of early warning models are the frequency ratio (FR) probability model, the stakeholder value (STV) cross-sectional model, and the subjective probability model. Owing to the low accuracy and poor objectivity of the previous early warning models, Kaminsky et al., (1998) constructed an early warning indicator system (KLR model) based on four aspects: indicator system, warning boundaries, data processing, and signal light display. Compared with the KLR model, the Probit model has better in-sample warning accuracy and less efficient out-of-sample risk warning (Berg \& Pattillo, 1999). Logit models have also received extensive attention from scholars who study risk early warning (Bussiere \& Fratzscher, 2006; Duca \& Peltonen, 2013; Gong et al., 2020). However, traditional risk warning models, including Logit and Probit models, often have low model warning efficiency and insufficient crisis identification ability because of linear assumptions and other prerequisites.

In recent years, nonlinear machine learning algorithms have been introduced into risk early warning research. Building and optimizing financial risk early warning models based on machine learning algorithms can help break the risk regulation bottleneck and improve the efficiency of risk early warning. For example, support vector machine (SVM) was introduced to explore risk management in financial markets (Groth \& Muntermann, 2011), and decision tree algorithms were used to build risk early warning models (Döpke et al., 2017). Moreover, Chatzis et al., (2018) compared the effectiveness of Logit, decision tree series, support vector machines, neural networks, and other techniques for systematic risk early warning, concluding that machine learning is more effective than traditional risk warning models and that training methods based on big data models lead to a significant increase in the accuracy of risk warnings.

While machine learning algorithms remain more efficient in terms of prediction in times of crisis, the predictive efficiency of daily stock market data during recessions can be improved by means of neuro-fuzzy modeling (Atsalakis et al., 2016). Beutel et al., (2019) compare the out-of-sample forecasting ability of different early warning models using a 45-year sample from banking systems in advanced economies, finding that machine learning algorithms are superior to 
Logit methods in out-of-sample forecasting. Bao et al., (2019) propose a strategy that combines unsupervised learning with supervised learning to perform credit risk assessment. The performance of the credit scoring model is improved by the hybrid use of machine learning algorithms. Machine learning algorithms could recognize various complex patterns. The application of machine learning algorithms to financial markets in developing countries is a very valuable area of research (Henrique et al., 2019). Machine learning algorithms have received increasingly more attention from scholars by virtue of their ability to recognize complex structures and efficient prediction capacity. There is also strong demand from industry and the government to build an intelligent risk early warning system. As such, building a risk control system based on machine learning algorithms is a new direction for risk early warning research in financial markets.

The main innovations and contributions of this paper are threefold. First, an AcF model is used to construct a dynamic measurement of tail risk in the Chinese capital market. The measurement provides a feasible solution to the model selection problem faced by the non-normal thick-tailed distribution of financial market return series. The measurement also more accurately portrays the characteristics of risk movements in various stages of the capital market and improves the efficiency of tail risk measurement in the Chinese capital market. Second, the adverse impact of the coordinated cross-market movement of tail risk on capital markets is explored from the perspective of strong tail risk spillovers and cross-market contagion. We find that the tail risk measure index has a crisis warning function and is a leading indicator of significant risks in financial markets. Third, we construct and optimize the tail risk early warning model of capital market based on machine learning algorithms. We use the capital market tail risk dynamic measurement index as the basis and pass the joint test of risk state research and crisis identification efficiency, which improves the crisis warning efficiency while enhancing the accuracy of risk state research and judgment. It is conducive to shortening the time lag of risk response of the regulator and promoting the development of capital market regulatory technology.

The rest of the paper is organized as follows. Section 2 introduces the tail risk measurement and early warning model construction. Section 3 reports and discusses the main empirical results in terms of dynamic measurements of tail risk in capital markets. Section 4 tests the validity and optimizes the capital market tail risk early warning model. Section 5 concludes the paper.

\section{Tail Risk Measurement and Early Warning Model Construction}

\subsection{Constructing Tail Risk Measurement Index Based on the ACF Model}

Extreme value theory overcomes the application problem of non-normal distributions of capital market return series. It achieves the effective measurement of the tail risk level under the condition of thick-tailed distributions of financial market return series. The AcF model minimizes information loss by modeling the 
dynamics of extreme observations in the tails of the distribution. Therefore, the $\mathrm{AcF}$ model is chosen to construct the capital market tail risk measurement index. The essence of the AcF model is extreme value modeling, and the first thing to determine before modeling is the extreme value series. When extreme value modeling the time series $\left\{Q_{t}\right\}$, the distribution function is as follows.

$$
\mathrm{H}(\mathrm{x} ; \mu, \sigma, \xi)=\exp \left(-\left(1+\xi \frac{x-\mu}{\sigma}\right)^{\frac{1}{\xi}}\right), \quad 1+\xi \frac{x-\mu}{\sigma}>0
$$

where $\mathrm{Q}_{t}$ is a univariate time series obtained based on a set of financial time series $\left\{X_{i t}\right\}_{i=1}^{p}$. In Eq. (1), $\mu, \sigma \in R, \mathrm{H}$ is referred to as the generalized extreme value (GEV) distribution, and $\xi$ is the shape parameter. When $\xi>0, \alpha=1 / \xi$ and $\mathrm{H}(\mathrm{x} ; \mu, \sigma, \xi)$ denotes the extreme value type II distribution; when $\xi=0, \mathrm{H}(\mathrm{x} ; \mu, \sigma, \xi)$ denotes the extreme value type I distribution; when $\xi<0, \mathrm{H}(\mathrm{x} ; \mu, \sigma, \xi)$ denotes the extreme value type III distribution. The capital market return series is characterized by spikes and thick tails, and the extreme value data taken from the capital market should obey the Fréchet distribution. The static Fréchet distribution is extended to a dynamic framework by means of parameter dynamization: $\left(\mu_{t}, \sigma_{t}, \alpha_{t}\right) \in \mathrm{F}_{t-1}=\sigma\left(Q_{t-1}, Q_{t-2}, \ldots\right)$, where $\alpha_{t}=1 / \xi_{t},\left\{\mathrm{Y}_{t}\right\}$ is a unit Frey hiatus random variable with independent identical distribution, $\left(\mu_{t}, \sigma_{t}, \alpha_{t}\right) \in \mathrm{F}_{t-1}=\sigma\left(Q_{t-1}, Q_{t-2}, \ldots\right)$.

For simplicity, assume that $\mu_{t}$ is a constant (a general approach in extreme value theory studies) (Zhao et al., 2018). The key variables in the modeling of the tail behavior are the scale and shape parameters, and the autoregressive structure is assigned to the time-varying parameters as follows in Eqs. (2) and (3).

$$
\begin{aligned}
& \log \sigma_{t}=\beta_{0}+\beta_{1} \log \sigma_{t-1}+\eta_{1}\left(Q_{t-1}\right) \\
& \log \alpha_{t}=\gamma_{0}+\gamma_{1} \log \sigma_{t-1}+\eta_{2}\left(Q_{t-1}\right)
\end{aligned}
$$

According to the clustering effect of extreme events, the previous period $\mathrm{Q}$ becomes larger, the volatility increases, and $\eta_{1}$ increases monotonically. The value of $\mathrm{Q}$ becomes larger, the thick tail $\alpha_{t}$ becomes smaller, and $\eta_{2}$ decreases monotonically. The autoregressive structure based on time-varying parameters shows that the AcF model implements a joint dynamic modeling of volatility and tail indices. Where $\eta()$ is an exponential function of the form $\rho_{0} \exp \left(-\rho_{1} \mathrm{x}\right)$ (continuous, differentiable, bounded; simple, flexible, intuitive, and invertible), and where $\rho_{1}>0$ and $\rho_{0}$ are used to control the monotonicity of the function, and the model is further simplified by Eqs. (4), (5), and (6).

$$
\begin{gathered}
Q_{t}=\mu+\sigma_{t} Y_{t}^{1 / \alpha_{t}}, \quad Y_{t} \sim F_{t-1}\left(\mu, \sigma_{t}, \alpha_{t}\right) \\
\log \sigma_{t}=\beta_{0}+\beta_{1} \log \sigma_{t-1}-\beta_{2} \exp \left(-\beta_{3} Q_{t-1}\right) \\
\log \alpha_{t}=\gamma_{0}+\gamma_{1} \log \sigma_{t-1}+\gamma_{2} \exp \left(-\gamma_{3} Q_{t-1}\right)
\end{gathered}
$$


The model parameters to be estimated are $\theta=\left(\beta_{0}, \beta_{1}, \beta_{3}, \gamma_{0}, \gamma_{1}, \gamma_{2}, \gamma_{3}, \mu\right)$. The parameter constraints are $\left\{\theta \mid \beta_{0}, \gamma_{0}, \mu \in \mathrm{R}, 0 \leq \beta_{1}, \gamma_{1}<1, \beta_{2}, \gamma_{2}, \beta_{3}, \gamma_{3}>0\right\}$, and the conditional density function of $Q_{t}$ is shown in Eq. (7).

$$
\mathrm{f}_{t}(\theta)=\mathrm{f}\left(\mathrm{Q}_{t} \mid \sigma_{t}, \alpha_{t}\right)=\alpha_{t} \sigma_{t}^{\alpha_{t}}\left(Q_{t}-\mu\right)^{-\left(\alpha_{t}+1\right)} \exp \left\{-\sigma_{t}^{\alpha_{t}}\left(Q_{t}-\mu\right)^{-\alpha_{t}}\right\}
$$

According to the conditional independence, the maximum likelihood function of the observation $\left\{Q_{t}\right\}_{i=1}^{n}$ is shown in Eq. (8).

$$
L_{n}(\theta)=\frac{1}{n} \sum_{t=1}^{n} l_{t}(\theta)=\frac{1}{n} \sum_{t=1}^{n}\left[\log \alpha_{t}+\alpha_{t} \log \sigma_{t}-\left(\alpha_{t}+1\right) \log \left(Q_{t}-\mu\right)-\sigma_{t}^{\alpha_{t}}\left(Q_{t}-\mu\right)^{-\alpha_{t}}\right]
$$

Given the initial parameter values of the optimization iteration $\theta=\left(\beta_{0}^{0}, \beta_{1}^{0}, \beta_{2}^{0}, \beta_{3}^{0}, \gamma_{0}^{0}, \gamma_{1}^{0}, \gamma_{2}^{0}, \gamma_{3}^{0}, \mu_{0}\right)$, and reasonable initial values $\left\{\alpha_{1}, \sigma_{1}\right\}$ for the iterative estimation, the tail risk measure index $\alpha_{t}$ can be estimated based on the parameter estimations.

\subsection{Building Tail Risk Warning Models Based on Machine Learning Algorithms}

Capital market tail risk generation and transmission paths are becoming increasingly complex. The dynamic evolution, nonlinear, and networked characteristics make the traditional static and linear models fail in capital market tail risk research and early warning. Machine learning algorithms have excellent prediction and model optimization capabilities, in part because of a superior ability to mine correlations between variables. Machine learning algorithms can analyze non-linear and complex problems, and are able to realize the effective research and early warning of capital market tail risk based on the full exploitation of data information. Machine learning algorithms are mainly divided into three types: supervised learning, unsupervised learning, and reinforcement learning. To realize capital market risk early warning, the risk level is first classified according to the unsupervised learning algorithm, and then the future risk state is judged by means of the supervised learning algorithm.

In this paper, we use Support Vector Machines (SVM), decision trees (CART, C50) and random forest (RF) algorithms to construct a capital market tail risk early warning model, and implement the algorithm training and optimization. Given the feature space sample set $D=\left\{\left(x_{1}, \alpha_{1}\right),\left(x_{2}, \alpha_{2}\right), \ldots\left(x_{n}, \alpha_{n}\right)\right\}$, the attribute set is $\mathrm{A}=\left\{y_{1}, y_{2}, \ldots, y_{d}\right\}$, where $\boldsymbol{x}_{\boldsymbol{i}} \in R^{n}, \alpha_{i} \in[0,1], y_{i} \in\{1,0\}, \mathrm{i}=1,2, \ldots, \mathrm{n}$. Where $\boldsymbol{x}_{\boldsymbol{i}}$ is the ith eigenvector of the risk state $i$, the $i$ th variable in the early warning indicator system, $m_{i}$ is the tail risk measure index, and $y_{i}$ is the classification marker. Assuming that the training data set is linearly divisible, the training set of the tail risk warning model is $T=\left\{\left(\boldsymbol{x}_{1}, y_{1}\right),\left(\boldsymbol{x}_{2}, y_{2}\right), \ldots\left(\boldsymbol{x}_{\boldsymbol{n}}, y_{n}\right)\right\}$.

\subsubsection{Support Vector Machine Early Warning Model}

The principle of SVM algorithm classification is interval maximization. By solving the convex quadratic programming (i.e., loss function minimization problem), the 
partition hyperplane of the feature space is found, enabling the achievement of effective classification and early warning. The rule for constructing the tail risk warning model based on the SVM algorithm is to solve the maximum partition hyperplane $\boldsymbol{\omega} * \boldsymbol{x}+b=0$. The geometric interval of the hyperplane about the sample points $\left(x_{i}\right.$, $\left.y_{i}\right)$ is defined as $\gamma_{i}=y_{i}\left(\frac{\boldsymbol{\omega}}{\|\boldsymbol{\omega}\|} * \boldsymbol{x}_{\boldsymbol{i}}+\frac{b}{\|\boldsymbol{\omega}\|}\right)$, and the distance from the hyperplane to all the sample points in the training set (i.e., the distance from the support vector to the hyperplane) is $\gamma=\min \sum_{i=1}^{n} \gamma_{i}$. Let $\omega=\frac{\omega}{\|\omega\| \gamma}$ and $b=\frac{b}{\|\omega\| \gamma}$. Then the SVM problem of solving the maximum partition hyperplane can be expressed as the following constrained optimization problem of solving $\omega$ and $b$.

$$
\max \sum \frac{1}{2}\|\omega\|^{2}, \quad \text { s.t. } y_{i}\left(\omega * x_{i}+b\right) \geq 1, i=1,2, \ldots n
$$

Construct the unconstrained Lagrangian function, where $\alpha_{i}$ is the Lagrangian multiplier and where $\alpha_{i} \geq 0$.

$$
L(\boldsymbol{\omega}, b, \boldsymbol{\alpha})=\frac{1}{2}\|\boldsymbol{\omega}\|^{2}-\sum_{i=1}^{n} \alpha_{i}\left(y_{i}\left(\boldsymbol{\omega} * \boldsymbol{x}_{i}+b\right)-1\right)
$$

Construct and solve the convex quadratic programming problem by selecting the appropriate kernel function $K(x, z)$ and penalty parameter $C>0$.

$$
\begin{gathered}
\min \frac{1}{2} \sum_{i=1}^{n} \sum_{j=1}^{n} \alpha_{i} \alpha_{j} y_{i} y_{j} K\left(x_{i}, x_{j}\right)-\sum_{i=1}^{n} \alpha_{i} \\
\text { s.t. } \sum_{i=1}^{n} \alpha_{i} y_{i}=0, \quad 0 \leq \alpha_{i} \leq C, i=1,2, \ldots, N
\end{gathered}
$$

The SVM classification decision function $\mathrm{f}(\mathrm{x})$ is obtained by optimally solving $\alpha^{*}$ and $b^{*}$, and the future risk state can be classified and predicted based on the classification decision function and the input variables.

$$
f(\boldsymbol{x})=\operatorname{sign}\left(\sum_{i=1}^{n} \alpha_{i}^{*} y_{i} K\left(\boldsymbol{x}, x_{i}\right)+b^{*}\right)
$$

\subsubsection{Decision Trees and Random Forest Early Warning Models}

A decision tree is essentially a conditional probability distribution model. It represents a mapping relationship between object attributes and object values. Decision trees of different shades correspond to probability models of different complexity. The tail risk warning model is constructed as a mapping from the early warning indicator system to the tail risk state of the market. By solving the optimization problem of loss function minimization, the decision tree algorithm generalizes a set of risk state classification rules with good generalization ability from the training 
Table 1 Decision tree algorithm optimal attribute division

\begin{tabular}{lll}
\hline Decision tree & Optimal attribute classification rules & Node selection \\
\hline ID3: Gain & $\begin{array}{ll}\operatorname{Ent}(T)=-\sum_{k=1}^{K} p_{k} \log _{2} p_{k} \\
\operatorname{Gain}(T, Y)=\operatorname{Ent}(T)-\sum_{v=1}^{V} \frac{\left|T^{v}\right|}{|T|} \operatorname{Ent}\left(T^{v}\right)\end{array}$ & Information gain value \\
C4.5: Gain.ratio & Gain.ratio $(T, y)=\frac{\operatorname{Gain}(T, y)}{-\sum_{v=1}^{V} \frac{\left|T^{v}\right|}{|T|} \log _{2} \frac{\left|T^{v}\right|}{|T|}}$ & Information gain rate \\
CART: Gini.index & Gini.index $(T, y)=\sum_{v=1}^{V} \frac{\left|T^{v}\right|}{|T|} \sum_{k=1}^{V} \sum_{k^{\prime} \neq k} p_{k} p_{k^{\prime}}$ & Gini coefficient \\
\hline
\end{tabular}

set. The tail risk warning model is constructed based on the decision tree by considering each variable in the warning index system as a characteristic attribute of the risk state.

The tree nodes are set and the risk state is judged by the selection of the optimal division attributes. A decision tree consisting of inner nodes (variables of the early warning indicator system), leaf nodes (attributes of risk states) and branches (probability calculation of risk state attribution) is generated by means of round-robin training, which is used to classify and judge the tail risk in the capital market. The division rules of the optimal division attribute of the decision tree mainly include information entropy, information gain, information gain ratio, and Gini coefficient. They correspond to different decision tree algorithms, and the optimal division rules of each decision tree algorithm are listed in Table 1.

Suppose the proportion of samples of class $\mathrm{k}$ in the current risk warning training set $\mathrm{T}$ is $p_{k}(k=1,2, \ldots,|K|)$. The discrete attribute $\mathrm{y}$ consists of $\mathrm{V}$ possible values $\left\{y^{1}, y^{2}, \ldots, y^{V}\right\}$. If $\mathrm{y}$ is used to partition the sample set $\mathrm{T}$, then $\mathrm{V}$ branch nodes are generated. The vth branch node then contains all samples in $\mathrm{T}$ that take the value $y^{v}$ on the attribute $y$, denoted as $T^{v}$. During the learning process of the decision tree algorithm, in order to improve the classification accuracy of the model, there may be too many branches in the decision tree because of the repetition of the number of divisions, thus causing an overfitting problem. Pruning is one of the methods to solve the overfitting problem. In addition, the integrated learning algorithm RF, a decision tree model based on the Bagging framework, improves the model prediction accuracy while solving the overfitting problem through algorithm integration.

\section{The Result of Tail Risk Measurement in Capital Market}

\subsection{Tail Risk Measure Index}

The non-normal distribution characteristic of financial market return series is an important reason for the low sensitivity of VaR family model risk measurements and risk lag measurements. Extreme value theory makes no assumptions of the specific form of the model and has a strong ability to foresee unexpected events. The theory thus has the dual advantage of accurate risk measurement and efficient risk warning. Therefore, we use the $\mathrm{AcF}$ model to construct tail risk measurement indices for the equity market and the bond market. 
Table 2 AcF parameter estimation results and t-test significance

\begin{tabular}{lllllllllll}
\hline Statistic & & $\beta_{0}$ & $\beta_{1}$ & $\beta_{2}$ & $\beta_{3}$ & $\gamma_{0}$ & $\gamma_{1}$ & $\gamma_{2}$ & $\gamma_{3}$ & $\mu$ \\
\hline Equity & Coef & 3.248 & 0.340 & 0.007 & 5.658 & 6.743 & 0.900 & 0.741 & 5.052 & -2.614 \\
& P value & 0.000 & 0.000 & 0.000 & 0.000 & 0.000 & 0.000 & 0.000 & 0.000 & 0.000 \\
Bond & Coef & 3.611 & 0.261 & 0.033 & 5.931 & 7.382 & 0.902 & 0.473 & 5.400 & -1.211 \\
& P value & 0.000 & 0.000 & 0.000 & 0.000 & 0.000 & 0.000 & 0.000 & 0.000 & 0.000 \\
\hline
\end{tabular}

We choose the spot CSI 300 index and the net interbank bond index (NIBI) as the proxy variables for the equity market and the bond market, respectively, to measure the tail risk of the Chinese capital market. After obtaining the five-minute highfrequency quotation series of the CSI 300 index, the maximum intra-day loss of the equity index is calculated in one-day blocks. The sample interval ranges from November 23, 2006 to May 25, 2020. Given the instability of the iterative algorithm at the initial point, the first 300 days are used as the model adaptation period. The five-minute high-frequency data of the CSI 300 Index have 48 data items per day, and the maximum loss of 48 data items on a given day is calculated to constitute the extreme loss series of the maximum intra-day loss of CSI 300 Index.

$$
\mathrm{Q}_{\text {return } 1}^{t}=\max \left\{- \text { return } 1_{i t}\right\}_{i=1}^{p}
$$

As in Eq. (14) above, the p-value of the CSI 300 index is taken as 48. Regarding the bond market data, we select the more liquid interbank bond aggregate index (net price) to exclude the effect of dividends to better reflect the real bond market sentiment. Given that the interbank bond market is less frequently traded than the equity market, the five-minute high-frequency data of the bond market are less valuable for our research objective. We calculate the five-day yield maximum loss by means of the moving window method, taking the current day and the previous four days as a calculation period, which constitutes the extreme value series of the bond market maximum loss within five days.

$$
\mathrm{Q}_{\text {return } 2}^{t}=\max \left\{- \text { return }_{i t}\right\}_{i=t-4}^{t}
$$

As in Eq. (15) above, $t$ represents the current period and $t-4$ denotes four trading days backward from the current period. From Eqs. (4), (5) and (6), the sequence of parameters to be estimated for the dynamic measure of capital market tail risk is $\theta=\left(\beta_{0}, \beta_{1}, \beta_{2}, \beta_{3}, \gamma_{0}, \gamma_{1}, \gamma_{2}, \gamma_{3}, \mu\right)$ with a total of nine parameters. Using the great likelihood estimation method with parameter constraints to estimate the parameters, the Matlab global optimization algorithm can be used to derive an optimal solution of the great likelihood estimation (see Table 2).

The results for the two markets show that the U.S. financial market is less noisy and has a stronger backward and forward tail risk dependence. For the Chinese capital market, the results of the constant term estimation are larger, which indicates that the autoregressive pattern of its risk trend operation is not obvious enough, and the market is more affected when facing shocks from uncertainties and the level of tail 

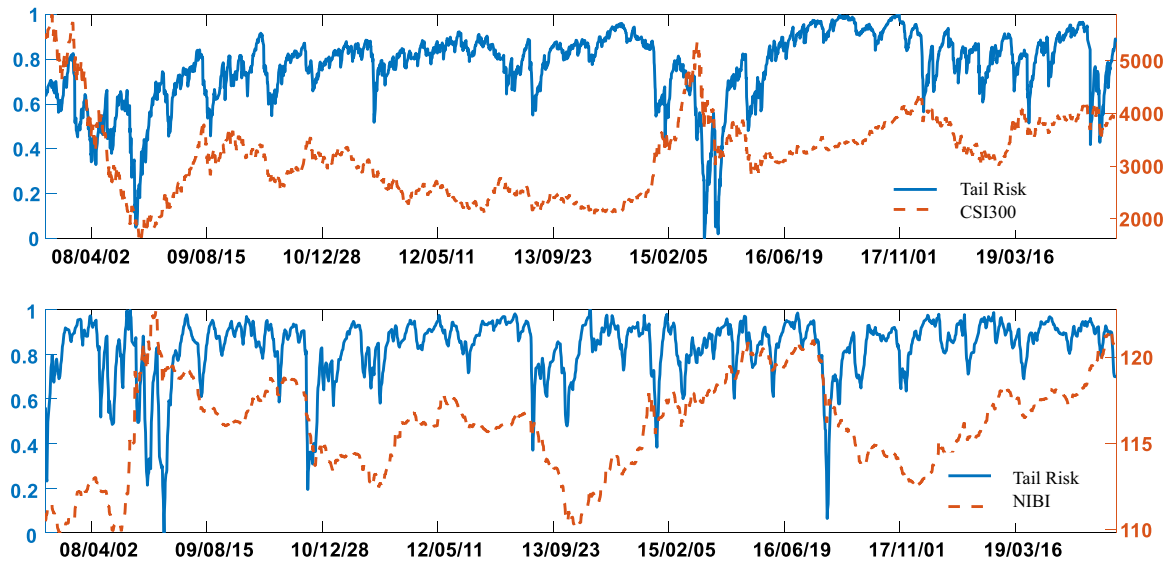

Fig. 1 Tail risk measurement index based on AcF model
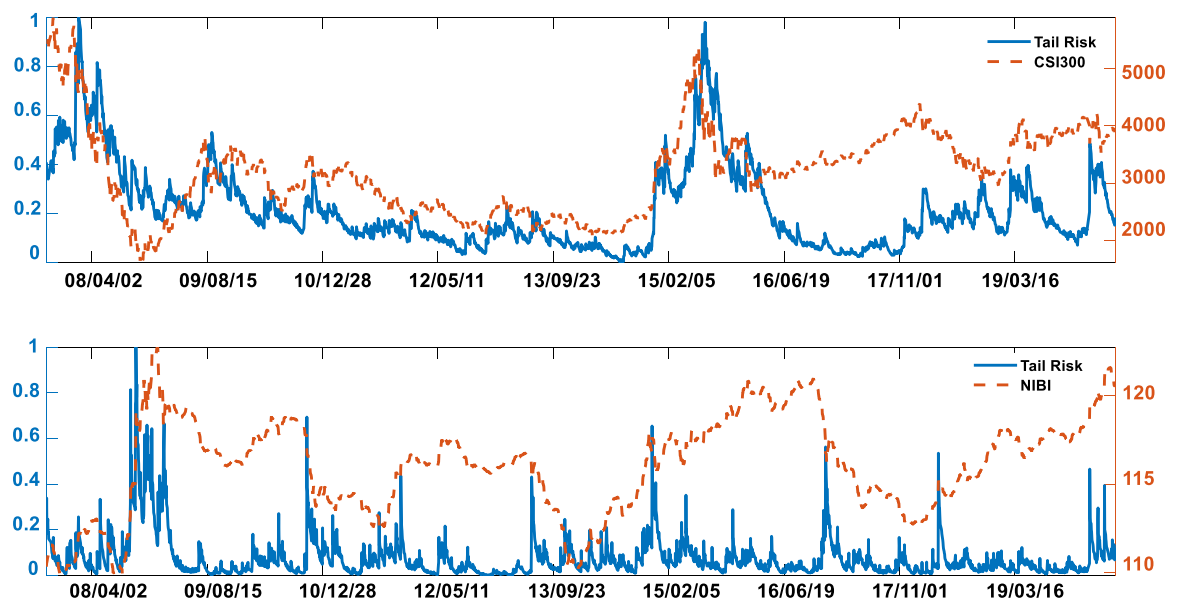

Fig. 2 Tail risk measure index based on GARCH-VaR model with 2 period time

risk is higher. To fully reflect the market risk trend, we normalize the tail risk index to $[0,1]$ according to the maximum-minimum method. Excluding the model adaptation period of 200 trading days, Fig. 1 shows the estimated results of the standardized tail risk index from September 18, 2007 to May 25, 2020.

The AcF model constructs a tail risk measure index, which corresponds to the economics of the inverse of the shape parameter of the extreme value type II Frechette distribution. Its theoretical range is $[0,+\infty]$. The larger the shape parameter (and the smaller the tail index), the thicker the tail and the greater the level of market tail risk. The change in the magnitude of the value of the tail index reflects the change in the magnitude of the level of tail risk in the capital market. In order to compare the efficiency of different models for tail risk measurement, we construct 
a GARCH-VaR model with 2 period time memory using the daily log returns of the same sample period, and we normalized the results of GARCH-VaR using the same method as AcF measurement. The model measurement results are shown in Fig. 2.

As illustrated in Figs. 1 and 2, the $\mathrm{AcF}$ model is more consistent than the GARCH-VaR model in measuring the equity market risk trend, which validates the applicability of the AcF model for measuring the tail risk trend in the Chinese capital market. Compared with the GARCH-VaR model, the AcF model is more sensitive when measuring the tail risk movements in the equity market. The equity market tail risk measurement index was based on the AcF model signaled market risk anomalies at the beginning of 2015, and the equity market risk level exceeded the historical high in May-June of 2015.

Based on the GARCH-VaR model, the two phases with the highest tail risk levels correspond to the 2008 subprime mortgage crisis period and the 2015 equity market crash period. However, the GARCH-VaR model fails to measure the tail risk in the bond market or accurately portray the absorption and release of tail risk in the bond market. Moreover, the GARCH-VaR model measures the risk inflection point of both the equity market and the bond market with some lagging effect. By contrast, the tail risk measure index constructed by the AcF model accurately portrays the volatile characteristics of the Chinese equity market during the U.S.-China trade war and the COVID-19 pandemic. Relatively, the AcF model is more effective both in terms of equity market risk measurement and the warning effect on the outbreak and tail risk.

In addition, Fig. 1 indicates how the bond market tail risk measure index based on the AcF model is continuous and smooth. There is no significant lagging effect on the movement of market risk trends. The bond market has a relatively high tail risk in the first half of 2013, the first half of 2015, and the second half of 2016. The observation corresponds to the biggest drop in the total CMB Interbank Bond Index since 2002 and the release of negative news such as the Federal Reserve interest rate hike in 2016, which led to several selling sprees in the Chinese bond market. Overall, the tail risk measurement index based on the AcF model not only has a significant ability to foresee extreme risks in the equity and bond markets, but its tail risk measurement efficiency is also significantly higher as compared to the GARCH-VaR model.

\subsection{Capital Market Tail Risk Synergy Campaign}

The study of capital market risk spillover and contagion effects is mainly divided into the mean spillover effect, the volatility spillover effect, the linear spillover effect, and the nonlinear spillover effect. Based on the dynamic measurement of tail risk, we discuss the cross-market synergistic movement of capital market tail risk divergence by comparing the upside and downside and tail risk spillover effects. First, we estimate MVGARCH-DCC models based on daily log returns and tail risk indices in equity as well as bond markets. Table 3 reports the results for the models with daily log returns. 
Table 3 Results of the MVGARCH-DCC model based on daily log returns for equity and bond markets

\begin{tabular}{|c|c|c|c|c|}
\hline \multicolumn{5}{|l|}{ Equity market } \\
\hline Statistic & Coef & Std. Error & z-Statistic & Prob \\
\hline L1.bond & 0.312 & 0.244 & 1.280 & 0.200 \\
\hline L1.ARCH & $0.059 * * *$ & 0.007 & 9.040 & 0.000 \\
\hline L1.GARCH & $0.940 * * *$ & 0.006 & 154.580 & 0.000 \\
\hline \multicolumn{5}{|l|}{ Bond market } \\
\hline Statistic & Coef & Std. Error & z-Statistic & Prob \\
\hline L1.bond & $0.478 * * *$ & 0.020 & 24.100 & 0.000 \\
\hline L1.ARCH & $0.197 * * *$ & 0.017 & 11.660 & 0.000 \\
\hline L1.GARCH & $0.817 * * *$ & 0.012 & 65.600 & 0.000 \\
\hline \multicolumn{5}{|l|}{ Adjustment DCC } \\
\hline Corr(equity,bond) & -0.030 & 0.020 & -1.480 & 0.139 \\
\hline$a$ & $0.101 * * *$ & 0.021 & 4.840 & 0.000 \\
\hline$b$ & $0.307 * *$ & 0.157 & 1.960 & 0.050 \\
\hline
\end{tabular}

As we report in Table 3, the mean equation of the daily log returns of the CSI 300 index and the total CMB interbank bond index is defined as follows in Eq. (16) and the variance equation is defined as follows in Eqs. (17) and (18).

$$
\begin{gathered}
\left(\begin{array}{l}
\text { stock }_{t} \\
\text { bond }_{t}
\end{array}\right)=\left(\begin{array}{c}
0.312 \\
0.478
\end{array}\right)\left(\text { bond }_{t-1}\right)+\left(\begin{array}{c}
\varepsilon_{1} \\
\varepsilon_{2}
\end{array}\right) \\
\sigma_{\text {stock }_{t}}^{2}=0.94 * \sigma_{\text {stock }_{t-1}}^{2}+0.059 * \varepsilon_{1, t-1}^{2} \\
\sigma_{\text {bond }_{t}}^{2}=0.817 * \sigma_{\text {bond }_{t-1}}^{2}+0.197 * \varepsilon_{2, t-1}^{2}
\end{gathered}
$$

By means of MVGARCH modeling, we have the conditional volatility of the equity market and bond market return series as defined by Eq. (19) for the DCC model.

$$
q_{i j t}=\rho_{i j t}(1-a-b)+a \varepsilon_{i, t-1} \varepsilon_{j, t-1}+b q_{i j, t-1}
$$

Based on the results of the MVGARCH-DCC model, the dynamic conditional correlation coefficients are significant and satisfy $a+b<1$, and the estimates are theoretically valid. Where $a$ denotes the degree of influence of the residuals of the MVGARCH model on the dynamic conditional correlation coefficients of the equity and bond markets (i.e., the degree of influence of new information on the dynamic correlation effect of the market), and $b$ denotes the degree of influence of historical market volatility correlation on the present (i.e., the degree of persistence of the correlation effect of market volatility). From the parameter estimates, the resonance 
Table 4 Results of the MVGARCH-DCC model based on the tail risk index for equity and bond markets Equity market tail risk

\begin{tabular}{|c|c|c|c|c|}
\hline Statistic & Coefficient & Std. Error & z-Statistic & Prob \\
\hline L1.equitytr & $0.99882 * * *$ & 0.00325 & 307.52000 & 0.00000 \\
\hline L1.bondtr & -0.00374 & 0.00280 & -1.33000 & 0.18200 \\
\hline cons & 0.00345 & 0.00326 & 1.06000 & 0.29000 \\
\hline L1.ARCH & $0.21905 * * *$ & 0.02803 & 7.81000 & 0.00000 \\
\hline L1.GARCH & $0.78273 * * *$ & 0.02397 & 32.65000 & 0.00000 \\
\hline cons & $0.00004 * * *$ & 0.00001 & 6.12000 & 0.00000 \\
\hline \multicolumn{5}{|c|}{ Bond market tail risk } \\
\hline Statistic & Coefficient & Std. Error & z-Statistic & Prob \\
\hline L1.equitytr & -0.00074 & 0.00156 & -0.48000 & 0.63400 \\
\hline L1.bondtr & $0.96662 * * *$ & 0.00346 & 279.19000 & 0.00000 \\
\hline cons & $0.03170 * * *$ & 0.00362 & 8.75000 & 0.00000 \\
\hline L1.ARCH & $0.40001 * * *$ & 0.04154 & 9.63000 & 0.00000 \\
\hline L1.GARCH & $0.59815 * * *$ & 0.03908 & 15.30000 & 0.00000 \\
\hline cons & $0.00001 * * *$ & 0.00000 & 5.92000 & 0.00000 \\
\hline \multicolumn{5}{|l|}{ Adjustment DCC } \\
\hline corr(equity,bond) & 0.02284 & 0.02141 & 1.07000 & 0.28600 \\
\hline$a$ & $0.02243 * *$ & 0.00921 & 2.44000 & 0.01500 \\
\hline$b$ & $0.85752 * * *$ & 0.06308 & 13.59000 & 0.00000 \\
\hline
\end{tabular}

effects of the equity and bond markets are weaker than the persistence of the historical correlation effects when facing new market shocks. The dynamic correlation coefficients of equity market and bond market volatility based on daily log returns are small, and the resonance effect of market volatility is not strong. Overall, the risk resonance coefficients of equity and bond markets when facing the same market disturbance are relatively small at only 0.101 .

Table 4 shows the results of the MVGARCH-DCC model constructed for the equity and bond market tail risk indices. We observe that the mean equation of the estimated MVGARCH-DCC model for the tail risk index of the equity and bond markets is given in Eq. (20) below, and the variance equation is given in Eqs. (21) and (22) below.

$$
\begin{aligned}
& \left(\begin{array}{l}
\text { stock }_{t} \\
\text { bond }_{t}
\end{array}\right)=\left(\begin{array}{l}
0.0345 \\
0.0317
\end{array}\right)+\left(\begin{array}{cc}
0.99882 & -0.00374 \\
-0.00074 & 0.96662
\end{array}\right)\left(\begin{array}{l}
\text { bond }_{t-1} \\
\text { stock }_{t-1}
\end{array}\right)+\left(\begin{array}{l}
\varepsilon_{1, t} \\
\varepsilon_{2, t}
\end{array}\right) \\
& \sigma_{\text {stock }}^{2}=0.00004+0.78273 * \sigma_{\text {stock }}^{2}+0.21905 * \varepsilon_{1, t-1}^{2} \\
& \sigma_{\text {bond }_{t}}^{2}=0.00001+0.59815 * \sigma_{\text {bond }_{t-1}}^{2}+0.40001 * \varepsilon_{2, t-1}^{2}
\end{aligned}
$$




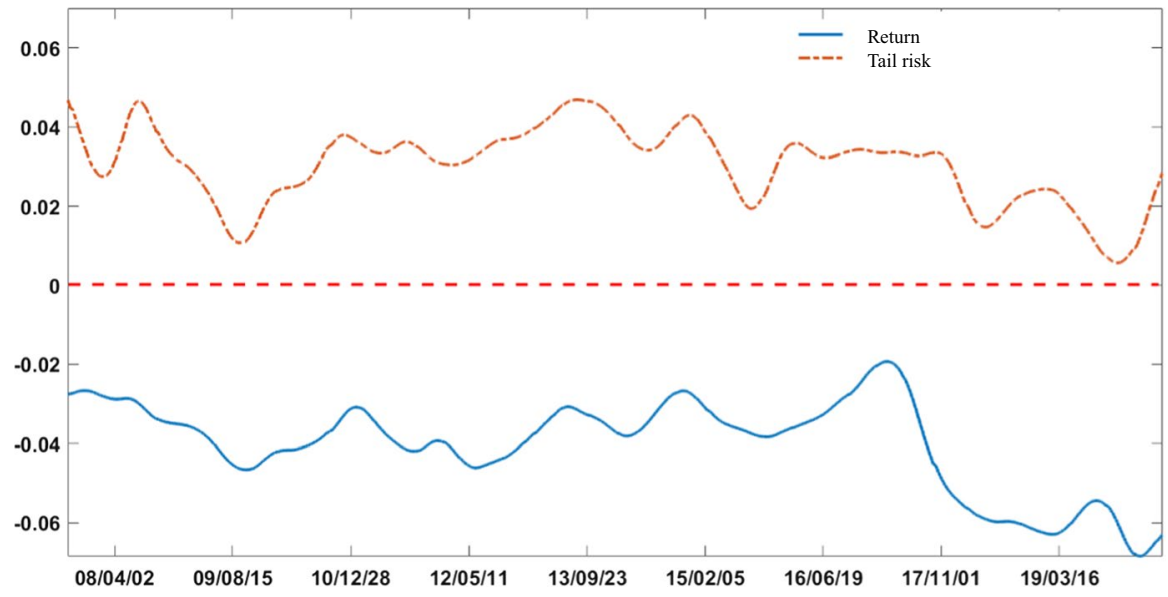

Fig. 3 HP filtered smoothing of risk resonance effects in stock market and bond market

Based on the results of the MVGARCH-DCC model, the dynamic conditional correlation coefficients are significant and satisfy $a+b<1$, and the model estimates are theoretically valid. From the results, the resonance effect of tail risk is also weaker than the persistence of the historical correlation effect in equity and bond markets when facing new market shocks. However, the overall mean value of the dynamic correlation effect of equity market and bond market risk is positive, which is in the opposite direction of the cross-market synergy effect of up and down.

Figure 3 illustrates the Hodrick-Prescott (HP) filtered trend of the dynamic correlation coefficients of tail risk and return series for the equity and bond markets. The trend of the dynamic conditional correlation coefficients of the return series and tail risk for the equity and bond markets are approximately the same. However, they are fundamentally different as the mean of the conditional correlation coefficient of return dynamics is less than 0 and the mean of dynamic correlation coefficient of tail risk is greater than 0. Furthermore, the tail risk synergies between the Chinese equity and bond markets were stronger than the yield synergies during the 2008 subprime mortgage crisis. Capital markets were exposed to common market shocks and external adverse conditions with high levels of systemic risk. From 2009 to 2015, the trend of cross-market synergistic movements of returns and tail risk is approximately the same. During the 2015 "equity market crash", China's equity markets suffered heavy losses while bond markets performed relatively stable. Based on the dynamic correlation coefficients of yields, the synergy between equity and bond market quotes is weak. During the COVID19 pandemic, the resonance effect between the equity market and the bond market was the weakest and reached the lowest value in history. However, the dynamic correlation coefficient of tail risk is warning the existence of significant tail risk 
spillover effects between equities and bonds, and the potential risk level of capital markets is high and must be strictly controlled to guard against a systemic crisis.

Overall, it is difficult to hedge tail risk in the equity and bond markets. Tail risk has a more persistent impact on the future risk state of capital markets. Relying on the resonance effect of upward and downward movements to build and adjust cross-market portfolios does not effectively diversify tail risk. Tail risk spillover and contagion are among the most important generators of capital market systemic risk, and tail risk is a leading indicator thereof.

\section{Testing and Optimizing the Validity of Capital Market Tail Risk Warning Models}

The tail risk measurement index based on the AcF model has a crisis warning function, and the coordinated movement of tail risk across markets is one of the main reasons for systemic risk generation in the capital market. Taking the Chinese capital market as an example, we construct a tail risk warning model based on a machine learning algorithm to improve the efficiency of tail risk warning in the capital market.

\subsection{Early Warning Indicator System}

The selection of the early warning index system is directly related to the efficiency of risk warning. We refer to the daily frequency risk early warning system constructed by Chatzis et al., (2018), the daily stock market quotation series of CSI 300 index up and down, CSI 300 index volume, CSI 300 index turnover and A-share market liquidity indicators are included in the stock market tail risk early warning indicator system. ${ }^{1}$ In addition, in order to study the impact of the macro and micro fundamentals of the financial market on the tail risk of the capital market, the early warning indicator system also includes the USD-RMB spot exchange rate, the money market term spread (SHIBOR-LIBOR overnight), the domestic and foreign funds borrowing spread (SHIBOR 1 year-1 week), and the interbank borrowing weighted rate ( 7 days). The bond market tail risk early warning model indicator system includes the rise and fall of the total CMB interbank bond index (net price), the Treasury maturity spread (difference between CMB Treasury yield to maturity: 5 years- 6 months), the bond market credit spread (difference between CMB corporate bond AAA grade and CMB Treasury yield to maturity: 6 months), the USDRMB spot exchange rate, the money market term spread (SHIBOR- LIBOR overnight), the domestic and foreign funds borrowing spread (SHIBOR 1 year-1 week), and the interbank borrowing weighted rate ( 7 days).

Indicators such as the capital market quotation series constitute the input indicators of the tail risk early warning indicator system, corresponding to the $i$ th feature

1 The construction of liquidity indicators is referred to Amihud (2002). 
vector $\boldsymbol{x}_{\boldsymbol{i}}$ of the risk state in the tail risk early warning model. $m_{i}$ is the tail risk measurement index based on the tail risk early warning model setting, $\boldsymbol{y}_{\boldsymbol{i}}$ is the classification marker, and the level of capital market tail risk above the alert line is coded as 1 and below the alert line as $0 . \boldsymbol{y}_{\boldsymbol{i}}$ is the output of the early warning model, which corresponds to the study of risk status.

Comparing the construction methods of risk early warning indexes, the scientific index method constructs early warning indices based on probability statistical theorem, which has higher scientific and validity compared with the critical value setting and reference system method. With reference to the scientific index method (Bai et al., 2020; Xu \& Chen, 2015), the capital market tail risk index is identified according to the dichotomous classification principle. Following the calculation principle, the measured tail risk index is subtracted from the mean value and divided by twice the standard deviation. 0.5 is used as the early warning line of the index, less than 0.5 is marked as 0 ; that is, the capital market tail risk is in the controllable range. More than 0.5 is marked as 1 ; that is, the capital market tail risk is higher in the crisis range. When the capital market tail risk is in the crisis zone, a risk warning signal is sent. The principle of constructing the output indicator of the early warning model is Eq. (15). There are 2647 observations marked as 0 and 433 observations marked as 1.2716 observations marked as 0 and 364 samples marked as 1 in the bond market tail risk warning indicator system.

$$
\text { Risk.sign }_{i}=\left\{\begin{array}{l}
1, \frac{m_{i}-\operatorname{mean}(m)}{2 * \operatorname{sd}(m)} \geq 0.5 \\
0, \frac{m_{i}-\operatorname{mean}(m)}{2 * \operatorname{sd}(m)}<0.5
\end{array}\right.
$$

\subsection{Early Warning Model Parameter Optimization and Validity Testing}

Based on the construction of the capital market tail risk early warning model and early warning indicator system, we use $85 \%$ of the data as the training set for model training and optimization, and $15 \%$ of the data as the test set for testing the out-ofsample early warning efficiency of the model. Given the obvious clustering effect of financial market volatility, financial crises and extreme risk events have small sample properties. Dividing the training and test sets according to the time axis, predetermining all samples into the same category during crisis and non-crisis periods tends to yield high overall warning efficiency, but the model's true crisis identification ability is extremely poor and the warning fails. To address the agglomerative small sample characteristics of crisis events and improve the robustness of the early warning model, we use a random sampling method to divide the model training set and model testing set. In addition, to verify the ability of the early warning model to identify crises, we use two methods to verify the model early warning efficiency based on the result confusion matrix of the early warning model. The first early warning efficiency is defined as $\mathrm{PE} 1=(\mathrm{TP}+\mathrm{TN}) /(\mathrm{TP}+\mathrm{TN}+\mathrm{FP}+\mathrm{FN})$, and the second early warning efficiency is defined as PE2 $=\mathrm{TP} /(\mathrm{TP}+\mathrm{FN})$. PE1 tests the 
Equity market
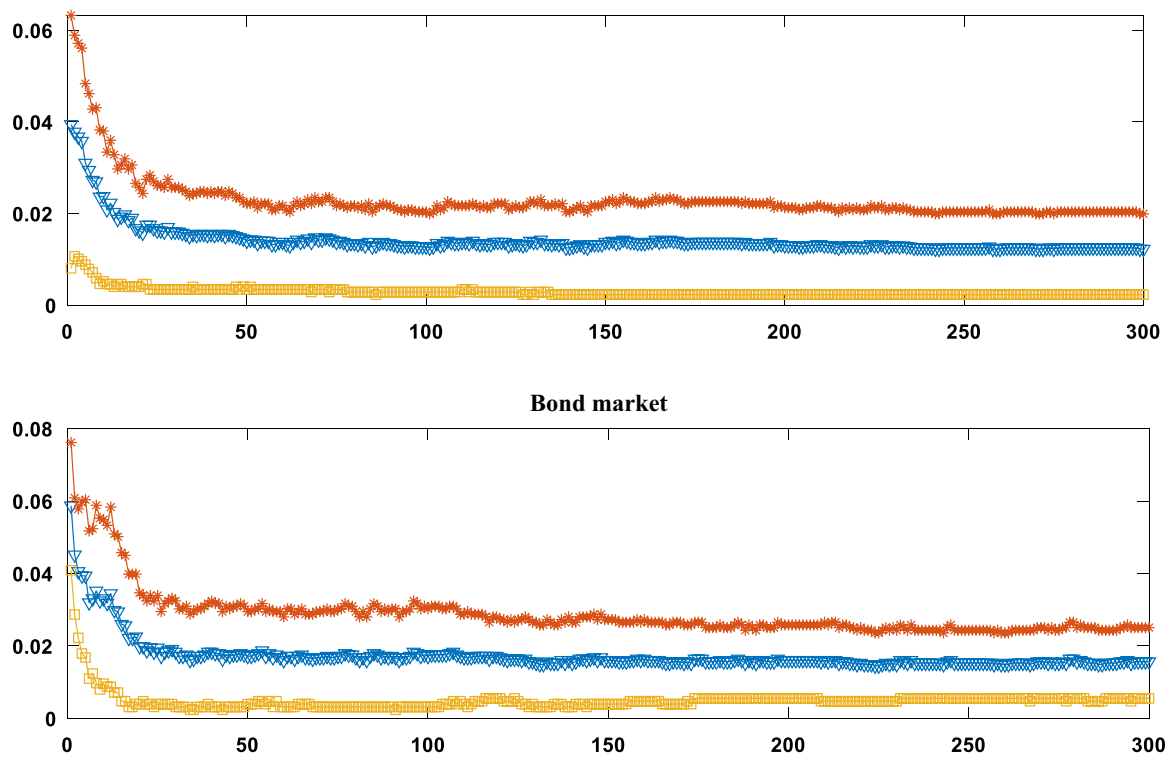

Fig. 4 Random Forest model training convergence

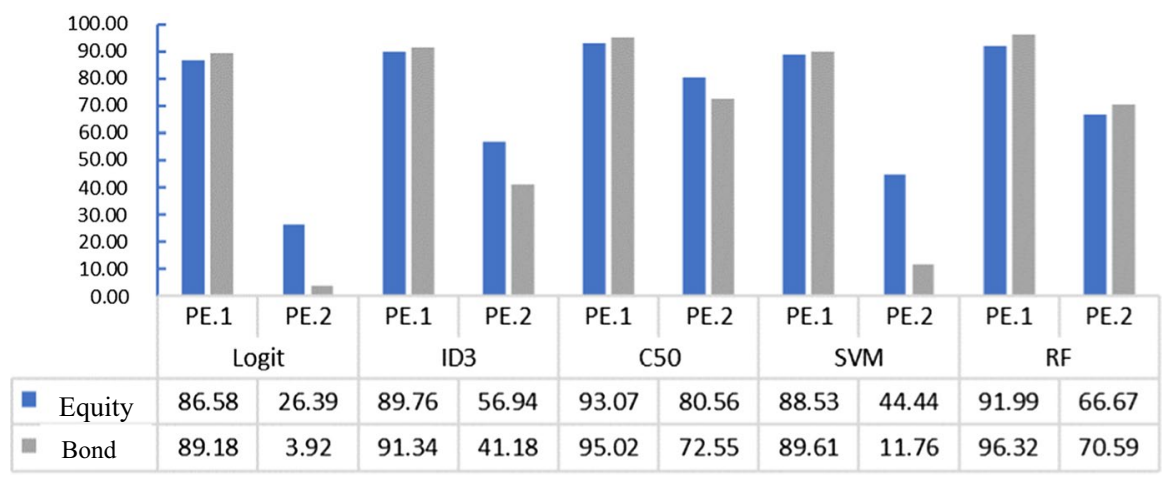

Fig. 5 Out-of-sample validity test of the early warning model (\%)

accuracy of the early warning model to study and judge the risk state, and PE2 tests the accuracy of the early warning model to judge whether a crisis appears.

The parameter optimization takes PE1 as the objective function, and the higher the value of PE1, the higher the efficiency of the model. We select the optimal decision tree warning model by optimizing the node selection and penalty function settings. The optimal SVM early warning model is selected by the kernel function (radial kernel function). The optimal RF model is selected by the adjustment of the number of attribute nodes and decision trees. Figure 4 illustrates the convergence trend of the random forest model training. The tail risk warning models based on the 
random forest algorithm all converge significantly after 150 decision trees. Therefore, the optimal number of decision trees included in the random forest is 150 , and the number of random samples as candidate variables is set to 2 and 3 for each split of the stock market and the bond market, respectively.

Figure 5 shows the results of the out-of-sample warning efficiency test for each warning model after parameter tuning. The ultimate purpose of constructing the early warning model is to accurately identify crises and inform capital market risk regulation. According to Fig. 5, the effectiveness of the Logit model in judging the future risk state is high, and the accuracy rates of judging the risk state of the stock market and bond market are $86.58 \%$ and $89.18 \%$, respectively. However, the crisis identification ability of the Logit model is extremely weak, and the accuracy rates of crisis identification for the stock market and the bond market are as low as $26.39 \%$ and $3.92 \%$, respectively. The Logit model-based capital market tail risk warning fails. Compared with the Logit model, the machine learning algorithm substantially improves the model out-of-sample warning efficiency, and the ID3, C50, SVM and $\mathrm{RF}$ algorithms are not only very accurate in determining the future risk state but also have strong crisis recognition ability. The accuracy rates of crisis recognition for the stock market are $56.94 \%, 80.56 \%, 44.44 \%$ and 66.67 , respectively. The accuracy rates of crisis recognition for the bond market are $41.18 \%, 72.55 \%, 11.76 \%$ and $70.59 \%$, respectively.

In addition, further comparison of PE2 of the SVM, ID3, C50 and RF algorithms shows that the crisis identification ability of different machine learning algorithms also differs. The efficiency of capital market tail risk warning based on the decision tree model is ahead of the support vector machine model. Although the PE2 of the ID3 algorithm and the C5.0 algorithm are higher than that of the integrated learning algorithm RF, the unpruned decision tree is likely to have overfitting problems. To further improve the crisis efficiency of the early warning model, we conduct further model optimization and testing.

\subsection{Optimization and Testing of Early Warning Models Based on Non-equilibrium Dataset Processing}

Based on the early warning indicator system, the rate of stock market tail risk exceeding the alert line is about $14.1 \%$, and the rate of bond market tail risk exceeding the alert line is about $11.8 \%$, which is a significant non-equilibrium dataset. To further improve the model warning efficiency and to test the robustness of the machine learning algorithm on the efficiency of capital market tail risk warning, we process the optimized early warning model based on the unbalanced dataset and test the optimized model early warning efficiency. There are four main methods for processing non-equilibrium datasets: (1) undersampling, (2) oversampling, (3) double sampling, and (4) ROSE synthetic sampling. Undersampling is no-release sampling, oversampling is with-release sampling, double sampling uses both methods, undersampling with no-release for large samples and oversampling with-release for small samples, and ROSE generates a balanced artificial data set by means of oversampling and balanced bootstrap. 
Table 5 Efficiency of tail risk warning based on the optimization of unbalanced dataset (\%)

\begin{tabular}{|c|c|c|c|c|c|c|}
\hline & & & Undersampling & Oversampling & Double sampling & ROSE \\
\hline \multirow[t]{10}{*}{ Equity } & \multirow[t]{2}{*}{ Logit } & PE.1 & 69.05 & 81.82 & 77.49 & 79.44 \\
\hline & & PE. 2 & 80.56 & 68.06 & 73.61 & 70.83 \\
\hline & \multirow[t]{2}{*}{ CART } & PE.1 & 80.09 & 86.58 & 85.06 & 88.10 \\
\hline & & PE. 2 & 88.89 & 84.72 & 80.56 & 66.67 \\
\hline & \multirow[t]{2}{*}{ C50 } & PE.1 & 83.77 & 94.16 & 94.16 & 87.01 \\
\hline & & PE. 2 & 98.61 & 83.33 & 84.72 & 69.44 \\
\hline & \multirow[t]{2}{*}{ SVM } & PE.1 & 75.54 & 88.96 & 88.74 & 86.80 \\
\hline & & PE. 2 & 87.50 & 81.94 & 83.33 & 66.67 \\
\hline & \multirow[t]{2}{*}{$\mathrm{RF}$} & PE.1 & 84.20 & 94.37 & 93.07 & 89.61 \\
\hline & & PE. 2 & 97.22 & 81.94 & 75.00 & 68.06 \\
\hline \multirow[t]{10}{*}{ Bond } & \multirow[t]{2}{*}{ Logit } & PE.1 & 63.85 & 75.97 & 70.13 & 71.43 \\
\hline & & PE. 2 & 62.75 & 54.90 & 56.86 & 58.82 \\
\hline & \multirow[t]{2}{*}{ CART } & PE.1 & 84.63 & 87.45 & 75.97 & 76.84 \\
\hline & & PE. 2 & 82.35 & 80.39 & 84.31 & 56.86 \\
\hline & \multirow[t]{2}{*}{$\mathrm{C} 50$} & PE.1 & 81.39 & 96.97 & 95.89 & 82.90 \\
\hline & & PE. 2 & 96.08 & 88.24 & 90.20 & 62.75 \\
\hline & \multirow[t]{2}{*}{ SVM } & PE.1 & 79.65 & 88.31 & 85.93 & 86.80 \\
\hline & & PE. 2 & 88.24 & 84.31 & 90.20 & 66.67 \\
\hline & \multirow[t]{2}{*}{$\mathrm{RF}$} & PE.1 & 88.10 & 96.75 & 94.81 & 85.50 \\
\hline & & PE. 2 & 96.08 & 80.39 & 90.20 & 66.67 \\
\hline
\end{tabular}

Table 5 shows the out-of-sample warning efficiency test for capital market tail risk based on the optimization of the unbalanced dataset. As reported in Table 5, the out-of-sample warning efficiency of the model optimized by the non-equilibrium dataset is significantly improved. The machine learning algorithms are highly efficient in both the judgment of future risk states and the identification of capital market crises, and the RF algorithm has the highest comprehensive early warning efficiency. The RF algorithm under the undersampling, oversampling and ROSE synthetic sampling methods has the highest accuracy in judging the future risk state of the stock market at $84.20 \%, 94.37 \%$, and $89.61 \%$, respectively. The RF algorithm has the highest accuracy rate of $97.22 \%$ for stock market crisis identification under the undersampling method. The RF algorithm has the highest accuracy for bond market crisis identification under the undersampling and double sampling methods with $96.08 \%$ and $90.20 \%$, respectively. In addition, we verified the robustness of the machine learning algorithm for warning tail risk using restructured random sampling to obtain a non-stationary data set, and the warning validity test is shown in Table 6.

As reported, the machine learning algorithm still has a high warning efficiency for capital market tail risk after restructuring random sampling, and the warning model has a robust and efficient warning capability. Following the combination of non-equilibrium data set processing and machine learning algorithms, we conclude that the optimal model pairing for equity market tail risk early warning is 
Table 6 Early warning model validity test based on recombinant random sampling (\%)

\begin{tabular}{|c|c|c|c|c|c|c|}
\hline & & & Undersampling & Oversampling & Double sampling & ROSE \\
\hline \multirow[t]{8}{*}{ Equity } & \multirow[t]{2}{*}{ ID3 } & PE.1 & 64.94 & 57.79 & 84.85 & 80.74 \\
\hline & & PE. 2 & 87.50 & 61.90 & 81.67 & 66.18 \\
\hline & \multirow[t]{2}{*}{ C50 } & PE.1 & 82.25 & 95.45 & 93.72 & 89.18 \\
\hline & & PE.2 & 98.44 & 84.13 & 86.67 & 73.53 \\
\hline & \multirow[t]{2}{*}{ SVM } & PE.1 & 83.81 & 88.74 & 89.18 & 87.08 \\
\hline & & PE.2 & 82.81 & 74.60 & 81.67 & 72.06 \\
\hline & \multirow[t]{2}{*}{$\mathrm{RF}$} & PE.1 & 81.82 & 94.59 & 93.72 & 91.13 \\
\hline & & PE. 2 & 96.88 & 82.54 & 83.33 & 70.59 \\
\hline \multirow[t]{8}{*}{ Bond } & \multirow[t]{2}{*}{ ID3 } & PE.1 & 79.65 & 83.98 & 83.77 & 93.94 \\
\hline & & PE. 2 & 91.07 & 82.14 & 83.67 & 81.63 \\
\hline & \multirow[t]{2}{*}{$\mathrm{C} 50$} & PE.1 & 86.58 & 96.10 & 94.59 & 81.82 \\
\hline & & PE. 2 & 100.00 & 78.57 & 93.88 & 62.00 \\
\hline & \multirow[t]{2}{*}{ SVM } & PE.1 & 79.00 & 84.63 & 84.63 & 85.06 \\
\hline & & PE. 2 & 83.93 & 64.29 & 83.67 & 54.00 \\
\hline & \multirow[t]{2}{*}{$\mathrm{RF}$} & PE.1 & 87.66 & 95.45 & 93.94 & 83.26 \\
\hline & & PE. 2 & 96.43 & 73.21 & 81.63 & 65.00 \\
\hline
\end{tabular}

oversampling-RF algorithm. The optimal model pairing for bond market tail risk early warning is double sampling-RF algorithm. Their out-of-sample risk state research and judgment accuracy is $94.37 \%$ and $94.81 \%$, respectively, and their outof-sample crisis warning accuracy is $81.94 \%$ and $90.20 \%$, respectively.

\section{Conclusion}

This paper addresses the insufficient ability of traditional econometric models to foresee extreme risks, long response time lag, and restricted conditions for model application. The AcF model is used to realize the dynamic measurement of tail risk in the Chinese capital market. We propose to construct and optimize a tail risk early warning model based on machine learning algorithms by using a tail risk measurement index. By means of dynamic tail risk measurement and analysis, we find that compared with traditional tail risk measurement methods, the AcF model based on dynamic extreme value theory addresses the challenges of non-normal distributions of financial time series data and the insufficient foreseeability of extreme risk events. The AcF model is highly responsive to abnormal volatility and tail risk events in financial markets, and the tail risk index based on the AcF model is an early indicator of capital market crises and major risks. The analysis of the cross-market synergy movement of capital markets shows that the tail risk synergy effect of capital markets is significantly stronger than the upside and downside synergy effect. The cross-market synergy of tail risk is particularly prominent in times of crisis, and relying solely on the analysis of upside and downside resonance effects to adjust cross-market portfolios does not effectively disperse tail risk. 
If the model is constructed and optimized based on the accuracy of risk state research, bias towards the judgment of risk-free state due to the small sample characteristics of crises is very likely, thus causing the failure of early warning. In this paper, we examine the out-of-sample warning efficiency of tail risk warning models based on machine learning algorithms by combining the accuracy of risk state diagnosis and the accuracy of crisis identification as the model construction and optimization criteria. Based on the construction and optimization of the capital market tail risk early warning model, we find that the Logit model has a high accuracy rate for risk state estimation, but its crisis identification ability fails. The tail risk early warning model based on SVM, ID3, C50 and RF algorithms can not only effectively research and judge the risk state but also accurately identify crises. The validity test of the early warning model with regrouping random sampling verifies the robustness of the tail risk early warning model based on machine learning algorithms for efficient warning. Via comprehensive model optimization and testing, the optimal model collocation for tail risk early warning in the stock market is oversamplingrandom forest algorithm. the optimal model collocation for tail risk early warning in the bond market is double sampling-random forest algorithm.

Acknowledgements Thanks are due to the Editor Professor and to anonymous reviewers for their helpful and constructive comments. Any errors are solely the authors' responsibility. Funding was provided by National Natural Science Foundation of China (Grant No. 7207030651).

Funding This study was funded by the National Natural Science Foundation of China under the project "Research on Systematic Risk Monitoring, Early Warning and Control in Chinese Capital Market Based on Machine Learning Algorithm Optimization" (7207030651).

Availability of data and materials Data come from Wind and CSMAR.

Code availability Software application and custom code in Matlab \& R. Custom code is available upon request.

\section{Declarations}

Conflict of interest All Authors declare that they have no conflict of interest.

Informed Consent Informed consent was obtained from all individual participants included in the study.

\section{References}

Adrian, T., \& Brunnermeier, M. (2016). CoVar. American Economic Review, 106(7), 1705-1741.

Amihud, Y. (2002). Illiquidity and stock returns: Cross-section and time-series effects. Journal of Financial Markets, 5(1), 31-56.

Atsalakis, G., Protopapadakis, E., \& Valavanis, K. (2016). Stock trend forecasting in turbulent market periods using neuro-fuzzy systems. Operational Research, 16(2), 245-269.

Bai, H., Liu, S., Luo, X., Liu, L., \& Hao, W. (2020). Research on the measurement and early warning of real estate market based systemic financial risk in China. Journal of Financial Research, 8, 54-73.

Bao, W., Ning, L., \& Kong, Y. (2019). Integration of unsupervised and supervised machine learning algorithms for credit risk assessment. Expert Systems with Application, 128, 301-315. 
Benoit, S., Colliard, J., Hurlin, C., et al. (2017). Where the risks lie: A survey on systemic risk. Review of Finance, 21(01), 109-152.

Berg, A., \& Pattillo, C. (1999). Predicting currency crises: The indicators approach and an alternative. Journal of International Money and Finance, 18(4), 561-586.

Beutel, J., List, S., \& Schweinitz, G. (2019). Does machine learning help us predict banking crises. Journal of Financial Stability, 45, 1-28.

Billio, M., Getmansky, M., Lo, A. W., et al. (2012). Econometric measures of connectedness and systemic risk in the finance and insurance sectors. Journal of Financial Economics, 104(03), 535-559.

Bussiere, M., \& Fratzscher, M. (2006). Towards a new early warning system of financial crises. Journal of International Money and Finance, 25(6), 953-973.

Chatzis, S., Siakoulis, V., Petropoulos, A., et al. (2018). Forecasting stock market crisis events using deep and statistical machine learning techniques. Expert Systems with Applications, 112, 353-371.

Döpke, J., Fritsche, U., \& Pierdzioch, C. (2017). Predicting recessions with boosted regression trees. International Journal of Forecasting, 33(4), 745-759.

Duca, M., \& Peltonen, T. (2013). Assessing systemic risks and predicting systemic events. Journal of Banking and Finance, 37(7), 2183-2195.

Embrechts, P., \& Schmidli, H. (1994). Modelling of extremal events in insurance and finance. Mathematical Methods of Operations Research, 39(1), 1-34.

Engle, R. (2002). Dynamic conditional correlation. Journal of Business and Economic Statistics, 20(3), 339-350.

Engle, R., \& Manganelli, S. (2004). CAViaR: Conditional autoregressive value at risk by regression quantiles. Journal of Business and Economic Statistics, 22(4), 367-381.

Feng, J. (2002). The research on the extremum VaR measurement of the risks of return ratio of Shanghai and Shenzhen stock market. Statistical Research, 4, 34-38.

Girardi, G., \& Ergün, A. (2013). Systemic risk measurement: Multivariate GARCH estimation of CoVaR. Journal of Banking and Finance, 37(8), 3169-3180.

Gong, X., Xiong, X., \& Zhang, W. (2020). Research on systemic risk measurement and spillover effect of financial institutions in China. Management World, 36(8), 65-83.

Groth, S., \& Muntermann, J. (2011). An intraday market risk management approach based on textual analysis. Decision Support Systems, 50(4), 680-691.

Henrique, B., Sobreiro, V., \& Kimura, H. (2019). Literature review: Machine learning techniques applied to financial market prediction. Expert Systems with Applications, 124, 226-251.

Jansen, D., \& De Vries, C. (1991). On the frequency of large stock returns: Putting booms and busts into perspective. Review of Economics and Statistics, 73(1), 18-24.

Kaminsky, G., Lizondo, S., \& Reinhart, C. (1998). Leading indicators of currency crises. IMF Staff Papers, 45(1), 1-48.

Li, C., Ma, W., \& Wang, B. (2010). Research on spillover effect within financial markets. The Journal of Quantitative \& Technical Economics, 27(6), 3-19.

Li, Y., Zhuang, X., Wang, J., \& Zhang, W. (2020). Analysis of the impact of Sino-US trade friction on China's stock market based on complex networks. The North American Journal of Economics and Finance, 52, 1-18.

Longin, F. (2000). Capital requirement: A new method based on extreme price variations. Journal of Risk Finance, 2(1), 42-50.

Massacci, D. (2017). Tail risk dynamics in stock returns: Links to the macroeconomy and global markets connectedness. Management Science, 63(9), 3072-3089.

McNeil, A. (1998). Calculating quantile risk measures for financial time series using extreme value theory. ASTIN Bulletin, 27, 117-137.

Wang, Y., \& Zhou, Y. (2012). The empirical study of China's stock market risk in extreme situations. Chinese Journal of Management Science, 20(3), 20-27.

Xu, D., \& Chen, S. (2015). a study on the measurement of systemic financial risk based on financial stress index. Economic Perspectives, 4, 69-78.

Xu, W., \& Huang, Y. (2008). Empirical analysis on GARCH-type models and VaR. The Journal of Quantitative \& Technical Economics, 1, 120-132.

Yang, Z., Chen, Y., \& Zhang, P. (2020). Macroeconomic shocks, financial risk transmission and governance responses under major public emergencies. Management World, 36(05), 13-35.

Zhao, Z., Zhang, Z., \& Chen, R. (2018). Modeling maxima with autoregressive conditional Fréchet model. Journal of Econometrics, 7(4), 325-351. 
Publisher's Note Springer Nature remains neutral with regard to jurisdictional claims in published maps and institutional affiliations. 